\title{
Sensordatenfusion zur Robusten und Präzisen EKF Lokalisierung von Mobilen Robotern
}

\author{
Kai O. Arras, Nicola Tomatis \\ Autonomous Systems Lab \\ Swiss Federal Institute of Technology Lausanne (EPFL) \\ $\mathrm{CH}-1015$ Lausanne \\ \{kai-oliver.arras, nicola.tomatis\}@epfl.ch
}

\begin{abstract}
Zusammenfassung: Diese Arbeit beschreibt einen Ansatz zur Lokalisierung von Mobilrobotern mittels der Kombination eines Laserscanners mit monokularem Video. Das Verfahren ist merkmalsbasiert und benutzt ein erweitertes Kalman filter (EKF) zur Datenfusion und Positionsschätzung. Die Umgebungsmerkmale sind Liniensegmente für den Laserscanner und vertikale Kanten für die Kamera. Physikalisch gut basierte Unsicherheitsmodelle beider Sensoren werden eingesetzt und bei Sensorkalibration und Merkmalsextraktion in Betracht gezogen. Dies liefert die geschätzten ersten zwei Momente der Merkmalsvektoren.

Die Experimente, die auf einem vollständig autonomen Roboter durchgeführt wurden, zielten auf zwei Fragestellungen ab: In welchem Mass kann das Hinzufügen videobasierter Umgebungsinformation die Navigation hinsichtlich Robustheit und Präzision verbessern? Die dazu ausgeführten Experimente zeigen, dass gerade in schwierigen Lokalisierungsszenarien wie lange Korridore, die Bildinformation einen unerlässlichen Beitrag liefert und in der Lage ist, die Positionsschätzung im allgemeinen und besonders in der Orientierung zu verbessern.
\end{abstract}

\section{Einleitung}

Merkmalsbasierte Ansätze zur Umgebungsmodellierung sind eine geeignete Wahl wenn präzise Navigation und kompakte Repräsentationen gefragt sind. Des weiteren besteht ihr Vorteil in der Möglichkeit, Merkmalsinformation verschiedenartiger Sensoren in analoger Weise direkt zu integrieren und für die Navigation nutzbar zu machen. Da dieser Ansatz aber nur bei fortlaufender Anwesenheit der gesuchten Merkmale funktioniert, ist es im Interesse robuster Navigation erstrebenswert, eine breitere Merkmalspalette einsetzen zu können.

Für die Fragestellung der Multisensorlokalisierung wird daher im Rahmen dieser Arbeit ein $360^{\circ}$-Laserscanner und ein monokulares Videosystem kombiniert. Die benutzten Merkmale sind horizontale Liniensegmente und vertikale Kanten. Sie werden auf Merkmalsebene durch das erweiterte Kalman Filter (EKF) fusioniert.

Das Lokalisierungsproblem von Mobilrobotern wurde schon früh mittels des EKF und Merkmalen aus Tiefendaten angegangen [8][9] und wird heute an vielen Orten eingesetzt, z.B. [6][1]. Vertikale Kanten in Kombination mit dem EKF wurden in [7] be- 
nutzt, in [10] wurde ein auf diesem Merkmal basierendes globales Relokalisierungsschema für das Problem eines in bekannter Umgebung verlorenen Roboters entwickelt.

Ein ähnlicher Multisensoraufbau wurde in [11] benutzt. Dort kommt ein Lasersensor zum Einsatz, welcher sowohl Tiefen-als auch Grauwertdaten liefert, jedoch nur in einem beschränkten Winkelbereich $\left(60^{\circ}\right)$ vor dem Roboter. Während ebenso vertikale Kanten als Videomerkmale zum Einsatz kommen, werden aus den Laserdaten keine Linien extrahiert, sondern rohe Tiefendaten einzeln zu modellierten Segmenten assoziiert. In einer kürzlich ausgeführten Arbeit [12] wurde die absolute Lokalisierungsgenauigkeit von Laser, monokularem und trinokularem Video mittels ground truth Messungen separat bestimmt und miteinander verglichen. Es wurde ähnliche Genauigkeit in allen drei Fällen festgestellt.

Diese Arbeit untersucht den Grad der Verbesserung hinsichtlich Robustheit und Präzision der Lokalisierung wenn zum Lasersensor videobasierte Information hinzugefügt wird. Wie in [11] werden die Unsicherheitsgrenzen der geschätzten Roboterposition untersucht, die unter der Annahme guter Modelle auch den Rückschluss auf die gesuchten ersten Momente zulassen. Aus diesem Grund wird versucht, physikalisch gut basierte Unsicherheitsmodelle für die eingesetzten Sensoren zu bekommen.

\section{Sensormodellierung}

Im Falle konsequent probabilistischer Sensordatenverarbeitung stellt sich die Frage nach korrekten Modellen der in den Messprozessen auftretenden Injektion von Unsicherheit. Streng genommen muss jede Quelle von Rauschen aufgespürt, modelliert und in genauer Kenntnis der Mess- und Verarbeitungsprozesse durchpropagiert werden. Dafür müssen diese Prozesse und der Sensor in allen konstruktiven Details bekannt sein. Es ist versucht worden so weit wie möglich gemäss dieser Überlegung vorzugehen.

Der hier eingesetzte Laserscanner ist ein Acuity AccuRange4000LIR mit einem Laufzeitprinzip. Der Sensor liefert analoge Signale für die Intensität des reflektierten Signals $i$ und eines für den Abstand $\rho$. Ersteres ist ein wichtiger Faktor, da er die Sicherheit der aktuellen Messung massgeblich mitbestimmt gemäss einer vom Messprinzip abhängenden Beziehung $\sigma_{\rho}=f(i)$. Bei weit entfernten oder schwach reflektierenden Oberflächen erhält man eine kleinere Signalstärke und damit eine unsicherere Distanzmessung. Bei Kenntnis der Intensität ist es somit möglich, ein physikalisch sehr gut basiertes Unsicherheitsmodell des Rauschens in radialer Richtung zu erlangen, das nicht nur der Distanz zum Objekt sondern auch seinen Oberflächeneigenschaften Rechnung trägt. In [2] wurden die dafür geeigneten Identifikationsexperimente durchgeführt, die zu einer einfachen, durch zwei Parameter beschreibbaren Beziehung $f($.$) ge-$ führt haben: $i_{\min }$ erlaubt das Verwerfen zu unsicherer Messungen mit $i<i_{\min }$ und $\sigma_{\rho}=0.4 \mathrm{~mm}$ wurde als konstante Standardabweichung für alle Messungen mit $i>i_{\text {min }}$ gefunden.

Beim verwendeten Videosystem handelt es sich um eine Pulnix TM-9701 full-frame grauwert-Kamera mit EIA Auflösung (640 x 480) und einem effektiven Öffnungswinkel von $54^{\circ}$. Da präzise geometrische Information aus den Bilddaten extrahiert wer- 
den soll, ist die Kalibration des Videosystems unerlässlich. Es wurde in [2] ein adequates Modell für das Linsensystem unter Berücksichtigung einiger vereinfachenden Faktoren gefunden und identifiziert mit insgesamt fünf Parametern für Bildzentrum $\left(c_{x}, c_{y}\right)$, Distortion $\left(k_{1}, k_{2}\right)$ und Fokallänge $C$. Extrinsische Parameter wurden durch eine Kameramontierung eliminiert, die Sensor- und Roboterkoordinatensystem in Übereinstimmung bringt. Unsicherheiten im Kalibrationsprozess (geometrische Unsicherheit des verwendeten Testmusters, Rauschen der Kameraelektronik) wurden gaussisch modelliert und propagiert, was neben den fünf Parametern des Kameramodells eine $5 \times 5$ - Koviarianzmatrix ergibt.

\section{Merkmalsextraktion}

Liniensegmente aus Laserscannerdaten werden mit dem Verfahren in [1] extrahiert. Es liefert die Schätzung der ersten beiden Momente, d.h. Mittelwertsvektor $[\alpha, r]^{T}$ und Kovarianzmatrix $C_{\alpha r}$ der Merkmalparameter $(\alpha, r)$ des Linienmodells

$$
\rho \cos (\varphi-\alpha)-r=0
$$

mit $(\varphi, \rho)$ als die in Polarkoordinaten vorliegenden Tiefendaten. Alle Operationen wie Assoziation und Zustandsschätzung im EKF werden im 2-dimensionalen $(\alpha, r)$-Raum ausgeführt. Somit liegen dem Filter die beiden ersten Merkmalsmomente $z_{l}=[\alpha, r]^{T}, R_{l}=C_{\alpha r}$ vor. Das Verfahren unterscheidet sich von der häufig eingesetzten, rekursiven split-and-merge-Technik, die auch in [8] und [6] benutzt wird: Für die Segmentierung kommt ein modelunabhängiges Kriterium auf der Basis von Punktgruppen zum Einsatz im Gegensatz zum linienspezifischen split-Kriterium auf der Basis eines Einzelpunktes. Die Assoziation von extrahierten Segmenten, die auf denselben physikalischen Objekten liegen, und deshalb im Interesse präziser Linienschätzung verschmolzen werden sollten, wird in [1] mit einem agglomerativen hierarchischen Clusteringverfahren mit einer Mahalanobis Distanzmatrix realisiert.

Vertikale Kanten werden mittels den folgenden vier Schritten gewonnen: spezialisierter Sobelfilter (nur x-Richtung), Verdünnung mit dynamischem Schwellwert (nur Kantenpixel von relativ maximalem Gradienten), horizontale Kantenbildkalibration und schliesslich Linienfit mit Kolonnen einer bestimmten Anzahl Pixel (der Fit vereinfacht sich zum 1D-Problem, Subpixelpräzision nach Kalibration). Rauschen der Kameraelektronik wird auf der Ebene des unkalibrierten Kantenbilds gaussisch modelliert und unter Berücksichtigung der unsicheren Kalibrationsparameter bei Kalibration und Kantenwinkelberechnung durchpropagiert. Dies resultiert im Wertepaar $z_{v}=\varphi$, $R_{v}=\sigma_{\varphi}^{2}$, das dem Kalman Filter zum Matching-Schritt übergeben wird.

\section{EKF Lokalisierung}

Als Folge der Sensordatenabstraktion bei merkmalsbasierten Ansätzen ist das Kalman Filter ohne Umweg mit Information mehrerer und andersartiger Sensoren erweiterbar. Es wird aus Platzgründen im folgenden darauf verzichtet, die Filtergleichungen in ihrer 
wohlbekannten Form wiederzugeben. Das Kalman Filter kann im Detail z.B. in [4] eingesehen werden oder im Kontext des Lokalisierungsproblems von Mobilrobotern in [9] oder [6]. Die vorliegende Anwendung des Filters bewegt sich ganz in diesem Rahmen.

Der Matching-Schritt bedarf jedoch aufgrund seiner Wichtigkeit und einiger Anpassungen an das vorliegende Problem weiterer Ausführung. Die korrekte Assozation von Prädiktionen und Beobachtungen, die während dieses Schrittes vollführt wird, ist von vitaler Bedeutung für die Konvergenz des Filters. Unter der (vernünftigen) Annahme, dass sowohl die Sensoren als auch die Merkmale untereinander durch statistisch unabhängige Fehlerquellen gestört sind, haben wir eine blockweise diagonale Beobachtungskovarianzmatrix $R$. Somit sind die Filtergleichungen merkmalsweise separierbar und es besteht die Freiheit, sie in einer für die Filterkonvergenz vorteilhaften Weise zu integrieren. Davon wird Gebrauch gemacht, indem die Merkmalsinformation der Sensoren in der Reihenfolge Laser-Video in das EKF integriert wird, da die Diskriminanz der Lasersegmente typischerweise höher ist als diejenige der vertikalen Kanten. Nach derselben Überlegung werden auch die einzelnen Prädiktions-Observations-paare gemäss dem Kriterium ihrer Qualität, iterativ, mit folgenden drei Schritten, integriert:

(i) Finden des momentan besten Zuordnungspaares

(ii) Schätzung

(iii) erneute Prädiktion aller noch nicht integrierten Beobachtungen.

Dasselbe Integrationsschema wurde auch in [11] und [12] benutzt, wo von gleichen Beobachtungen bezüglich Merkmalsdiskriminanz berichtet wird.

Die Qualität eines Paares aus Prädiktion $\hat{z}_{l, v}^{[j]}$ und Beobachtung $z_{l, v}^{[i]}$ bestimmt sich unterschiedlich für beide Sensoren:

- Für Liniensegmente ist das Qualitätskriterium eines Zuordnungspaares das der kleinsten Beobachtungsunsicherheit - nicht kleinste Mahalanobisdistanz wie in [11] oder [12]. Damit wird Robustheit gegenüber kleinen und unsicheren Segmenten erreicht. Das momentan beste Zuordnungspaar $\left(z_{l}^{[i]}, \hat{z}_{l}^{[j]}\right)$ ist demnach jenes von $z_{l}^{[i]}$, wofür gilt $\operatorname{trace}\left(R_{l}^{[i]}\right)=\min _{i}$ und das den validation-test

$$
\left(z_{l}^{[i]}-\hat{z}_{l}^{[j]}\right) S_{i j}^{-1}\left(z_{l}^{[i]}-\hat{z}_{l}^{[j]}\right)^{T} \leq \chi_{\alpha, n}^{2}
$$

besteht, wobei $S_{i j}$ die Innovationskovarianzmatrix des Paares ist und $\chi_{\alpha, n}^{2}$ ein einer $\chi^{2}$-Verteilung mit $n=2$ Freiheitsgraden entnommenem Wert entspricht, mit dem die Hypothese der Paarkorrektheit auf einem $\alpha$-Niveau verworfen wird.

- Das Kriterium für vertikale Kanten ist wie in [11] und [12] Eineindeutigkeit. Prädiktionen $\hat{z}_{v}^{[j]}$ in deren validation gates nur eine einzige Beobachtung $z_{v}^{[i]}$ liegt, werden bevorzugt und gemäss kleinster Mahalanobisdistanz integriert, sofern sie Gleichung 2 mit $n=1$ erfüllen (Subskripts $l$ werden zu $v$ ). Wenn keine eindeutigen Zuordnungen mehr existieren, werden auch Paare mit mehreren Beobachtungen im validation gate berücksichtigt, indem die der Prädiktion nächstliegende Beobachtung akzeptiert wird. Dies ist motiviert durch das häufige Auftreten von Gruppen eng beieinander liegender vertikaler Kanten, deren Zuordnung besonders im Falle relativ grosser validation gates schwierig ist. 


\section{Implementation und Experimente}

\subsection{Der Roboter}

Die Experimentierplatform ist der Roboter Pygmalion, der kürzlich in unserem Labor entworfen und konstruiert wurde (Fig. 1). Seine Designprinzipien richteten sich nach den Anforderungen an einen Serviceroboter, wobei als Hauptkriterien hoher Autonomiegrad, freundliche Erscheinung und Sicherheit zur Geltung kamen. Mit Abmessungen von $45 \times 45 \times 70 \mathrm{~cm}$ und einem Gewicht von ca. $55 \mathrm{~kg}$ ist er von moderater Grösse und Gefahr verglichen mit Vehikeln der selben Leistungsklasse. Auf dem Roboter läuft das harte Echtzeitbetriebssystem XOberon [5].

\subsection{Experimente}

Lange Korridore stellen unabhängig von der Lokalisierungsmethode eine Herausforderung für mobile Roboter dar, einerseits weil sie in Anwendungsszenarien von Servicerobotern regelmässig vorkommen und andererseits weil sie die Schwierigkeit enthalten, in Korridorrichtung lokalisiert zu bleiben. Um die Leistung des unter Betracht stehenden Multisensorsystems unter diesen Bedingungen $\mathrm{zu}$ untersuchen wurden auf der 78 m-Testtrajektorie von Figur 3 zwei Experimente durchgeführt, eines zum Aspekt der Robustheit und eines zum Aspekt der Präzision. Der Roboter legte dabei eine Gesamtstrecke von mehr als $1 \mathrm{~km}$ zurück. Beide Experimente wurden in einem Schritt-fürSchritt Modus durchgeführt, mit dem auf dem Roboter laufenden Positionsregler für nicht-holonome Vehikel [3]. Eine lokale Navigationsstrategie für unmodellierte Objekte war nicht aktiv. Da der Lasersensor seine Winkelkalibration bei jedem Systemstart mittels Messung der vier vertikalen Profile ausführt (siehe Figur 1), besteht eine Winkelunsicherheit in der Roboter-zu-Sensor Koordinatentransformation. Dieser wird in der Prädiktion der Merkmalsunsicherheit zusätzlich Rechnung getragen.

Im ersten Experiment wurde die zuvor von Hand gemessene a priori Karte dahingehend modifiziert, dass im Korridor keine Segmentinformation vorkommt, die eine Aktualisierung der Roboterposition in Fahrtrichtung durch den Lasersensor erlaubt. Davon sind die senkrecht zum Korridor stehenden kurzen Schrankseiten betroffen. Dann fuhr der Roboter die Trajektorie drei mal ab unter ausschliesslicher Benutzung des Lasersensors. Drei weitere Fahrten wurden daraufhin mit dem Multisensorsystem gemacht. 

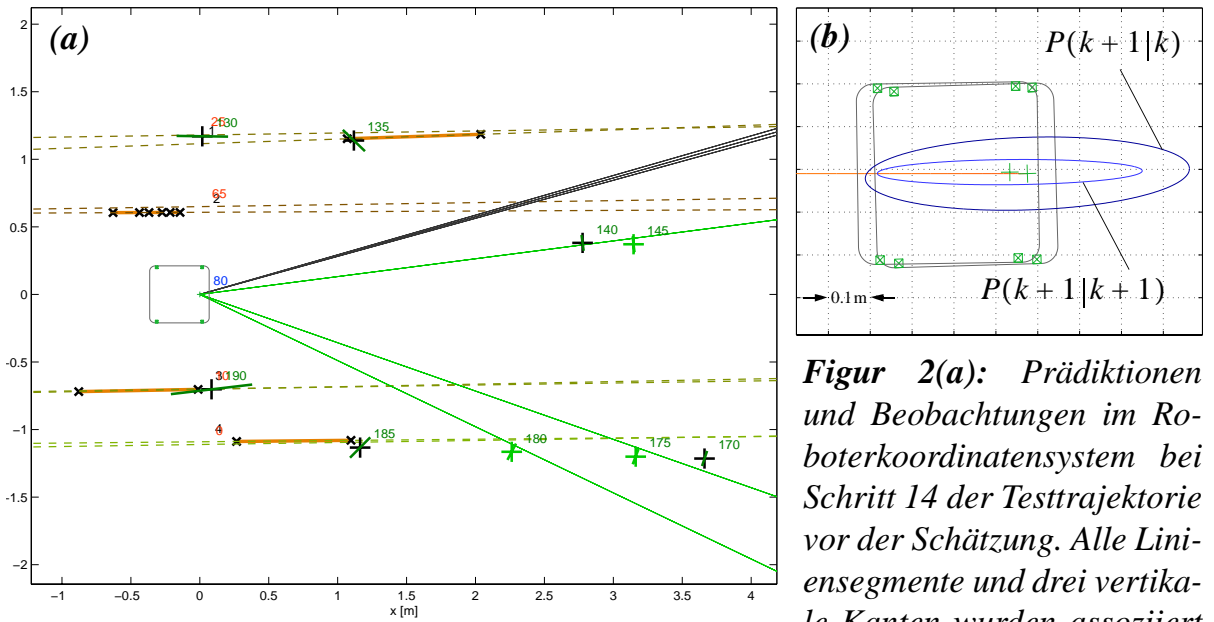

und Beobachtungen im Roboterkoordinatensystem bei Schritt 14 der Testtrajektorie vor der Schätzung. Alle Liniensegmente und drei vertikale Kanten wurden assoziiert (zu den Prädiktionen 145, 175, 180). (b) Positionsaktualisierung durch die Assoziationen von Figur (a). Das Kalman Filter korrigiert den Roboter ca. 5 cm nach rückwärts, was zu einer guten Übereinstimmung von Beobachtung und Prädiktion der drei assoziierten vertikalen Kanten führt. Dies demonstriert die Fähigkeit des Kalman Filters mit der Winkelinformation der vertikalen Kanten, auch in der in diesem Szenario kritischen Korridorrichtung zu korrigieren; die a posteriori Zustandskovarianzmatrix $P(k+1 \mid k+1)$ bleibt begrenzt.

Mit dem zweiten Experiment wurde der Einfluss des Videosystems auf die Unsicherheit der Positionsschätzung untersucht. Die Trajektorie wurde zehn mal abgefahren, fünf Versuche ohne Video, fünf Versuche mit. Die Karte war unmodifiziert, d.h. alle extrahierbaren Segmente wurden modelliert. Die Durchschnittswerte der frontalen, lateralen und azimutalen Unsicherheit wurden ermittelt und sind in Figur 4 dargestellt.

\subsection{Resultate}

Reproduzierbar waren im ersteren Fall des ersten Experiments (nur Laser) die Unsicherheiten und Schätzfehler in Fahrtrichtung am unteren Korridorende so gross, dass falsche Assoziationen zur Filterdivergenz geführt haben. Der Roboter war verloren (Figur 3a). Beim Hinzufügen der Information des Videosystems kann das Wachstum des Schätzfehlers in Fahrtrichtung begrenzt werden. In Figur 2 ist Schritt 14 der Trajektorie mit Prädiktionen und Beobachtungen beider Sensoren sowie der resultierenden Roboterschätzung abgebildet. Es verdeutlicht, wie das Filter in Abhängigkeit der vorliegenden Positionsunsicherheit - d.h. nach Integration der Lasersegmente - die Winkelinformation der vertikalen Kanten in kartesische Korrekturen der Roboterposition umwandelt. Die Korrekturen am unteren und oberen Korridorende waren unkritisch; der Roboter konnte seine Aufgabe jedesmal erfüllen und sicher zum Ausgangspunkt zurückkehren (Figur 3b).

Aus dem zweiten Experiment geht primär eine hohe absolute Lokalisierungsgenauigkeit hervor (Figur 4). Es kann beim Hinzufügen der Videoinformation eine allgemei- 

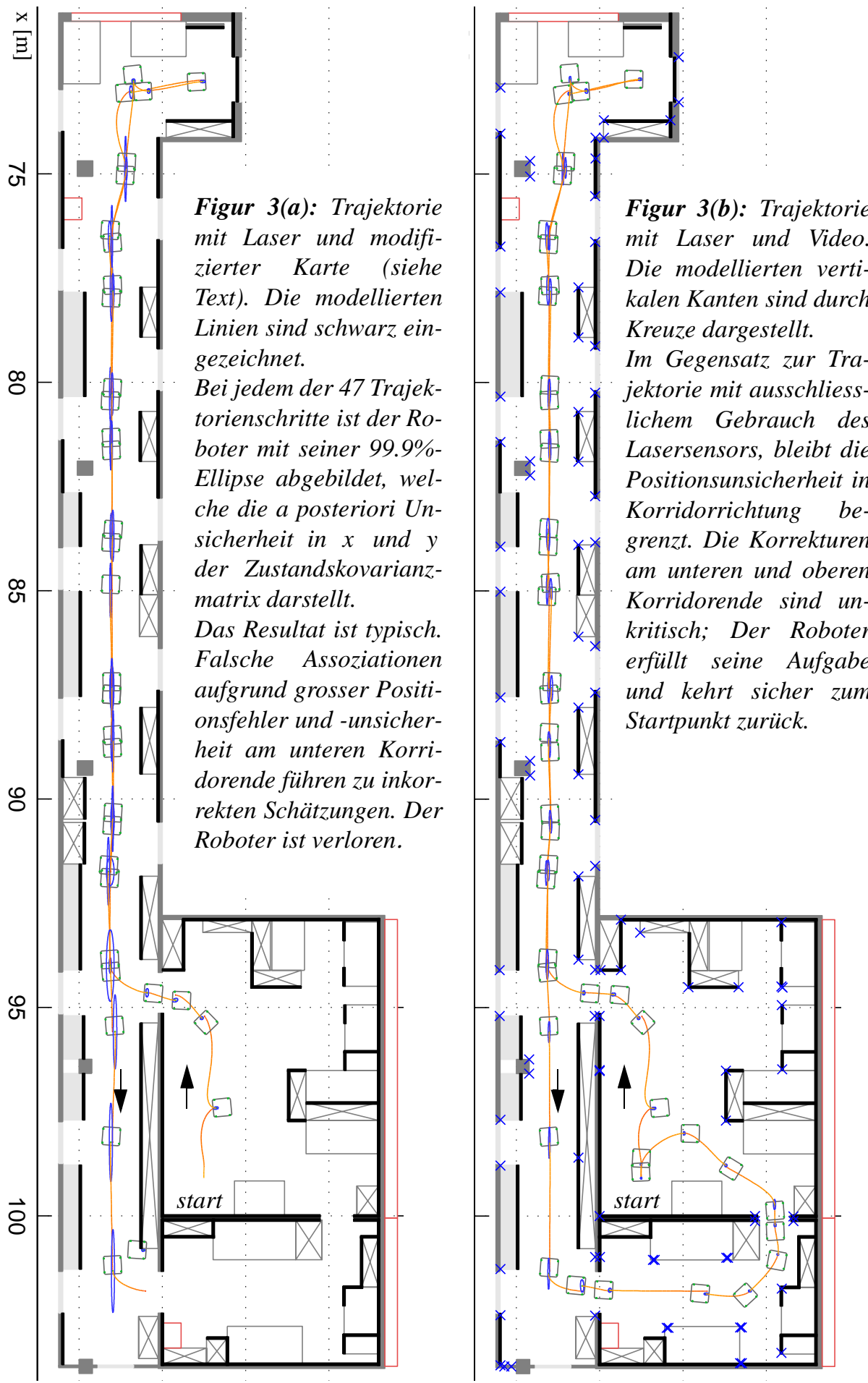

Autonome Mobile Systeme 1999, 15. Fachgespräch 

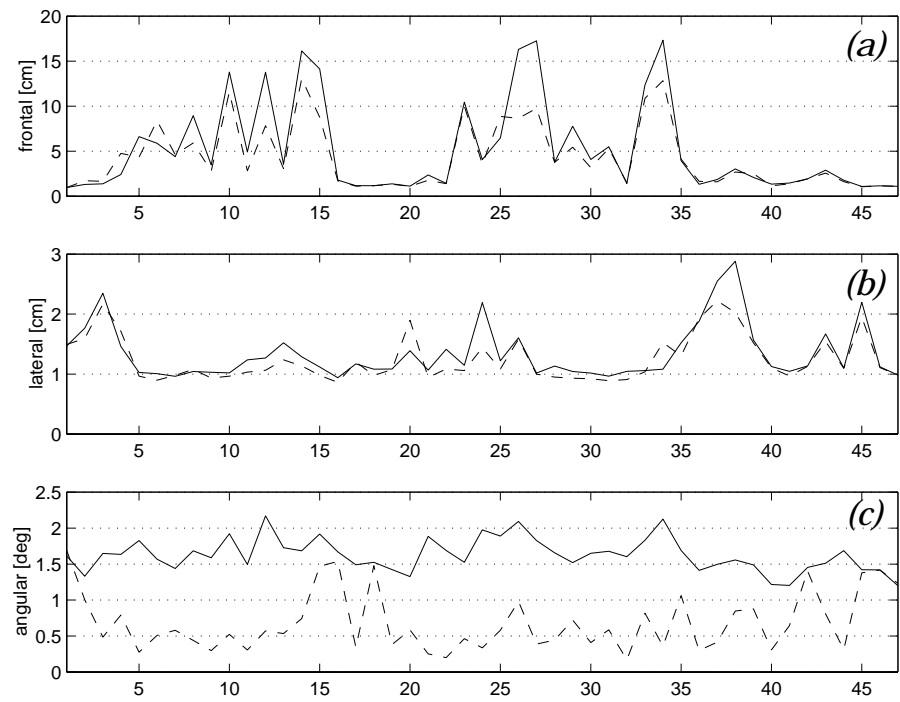

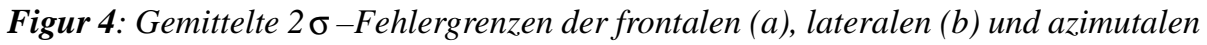
(c) a posteriori Unsicherheit bei den 47 Schritten der Testtrajektorie von Figur 3. Jeweils fünf Testläufe in beiden Modi wurden durchgeführt. Ausgezogene Linien: nur Lasersensor, gestrichelte Linien: Laser und Video.

ne Unsicherheitsreduktion gegenüber dem Laser-Modus festgestellt werden, obwohl die gemittelte Anzahl assoziierter vertikaler Kanten $\bar{n}_{v}$ (siehe Tabelle 1) gering ist. Auffallend ist die deutliche Unsicherheitsverringerung in der Orientierung (Figur 4c).

In Tabelle 1 sind ausserdem die über alles gemittelten Werte gegeben. Die Zykluszeiten wiederspiegeln Bruttorechenzeiten der eingesetzten PowerPC VME-Karte bei $300 \mathrm{MHz}$, auf der alle Berechnungen ausgeführt werden und enthalten ebenso die Zeiten von Datenakquisition, Low-Level Regler und Kommunikation.

\subsection{Diskussion}

In einigen Fällen von Figur 4 gibt es, wider der Erwartung, grössere Unsicherheiten des Multisensor-Modus gegenüber dem Laser-Modus. Dies ist möglich, da es sich um gemittelte Werte handelt, in denen Rauschen auf dem Niveau der assoziierten Merkmale desselben Ortes enthalten ist. So kann es z.B. sein, dass zwei senkrecht zueinander stehende Lasersegmente eine zuverlässigere Positionsschätzung erlauben als ein am gleichen Ort im Multisensor-Modus extrahiertes und assoziertes Liniensegment in Kombination mit zwei vertikalen Kanten.

Erfahrung mit früheren on-the-fly-Implementationen (fortlaufende Lokalisierung während der Fahrt) zeigen [1], dass die hier ausgeführte Schritt-für-Schritt Navigation ungünstig ist. Obwohl es bei ersterem einige zusätzliche Probleme zu lösen gibt, bringt eine fortlaufende Lokalisierung die Aktualisierungsrate in ein wesentlich besseres Verhältnis zur Dynamik der Filtervariablen und erhöht damit die Robustheit gegenüber wichtigen Faktoren wie eine unzulänglich kalibrierte Odometrie. 


\begin{tabular}{c|c|c}
\hline & Laser & Laser und Video \\
\hline \hline $2 \sigma_{\text {frontal }}$ & $5.191 \mathrm{~cm}$ & $4.258 \mathrm{~cm}$ \\
\hline $2 \sigma_{\text {lateral }}$ & $1.341 \mathrm{~cm}$ & $1.245 \mathrm{~cm}$ \\
\hline $2 \sigma_{\text {azimutal }}$ & $1.625^{\circ}$ & $0.687^{\circ}$ \\
\hline $\bar{n}_{l} / \bar{n}_{v}$ & $3.53 /-$ & $3.51 / 1.91$ \\
\hline$\overline{t_{\text {exe }}}$ & $30 \mathrm{~ms}$ & $330 \mathrm{~ms}$ \\
\hline
\end{tabular}

Tabelle 1: Gemittelte Werte der Fehlergrenzen, Anzahl assoziierter Liniensegmente $n_{l}$ und assoziierter vertikaler Kanten $n_{v}$, sowie die gemittelte Lokalisierungszyklusdauer $\overline{t_{\text {exe }}}$ bei voller Prozessorlast.

Die Assoziation von vertikalen Kanten ist aufgrund ihres Auftretens in Gruppen besonders fehleranfällig. Bei Türrahmen z.B. werden oft mehrere, eng beieinanderliegende Kanten extrahiert, da Schatten an Fugen und Kanten des Rahmens Evidenz für die Merkmalsextraktion erzeugen. Mit grossen validation gates aufgrund unsicherer Positionsprädiktionen wurden gelegentlich falsche Assoziationen produziert. Die Wirkung dieser fehlerhaften Zuordnungen bleibt aber gering, da diese Gruppen typischerweise recht kompakt sind. Eine Verbesserungsmöglichkeit ist der Versuch, nicht einzelne Beobachtungen mit einzelnen Prädiktionen zu assoziieren, sondern durch Ausnutzen ihrer räumlichen Beziehung, die von der a priori Karte bekannt ist, Konstellationen von Bebachtungen als Ganzes zuzuordnen. Dieses Problem wurde in [6] und [10] angegangen.

\section{Zusammenfassung und Ausblick}

Im Rahmen dieser Arbeit wurde der Beitrag video-basierter Umgebungsinformation zur robusten und präzisen Navigation von mobilen Robotern untersucht. Ein Lasersensor, der horizontale Liniensegmente als Merkmale für die Lokalisierung liefert, wurde mit einem monokularen Videosystem, welches vertikale Kanten extrahiert, kombiniert. Das erweiterte Kalman Filter wurde zur Datenfusion und Positionsschätzung eingesetzt.

Aus den Resultaten der Experimente ist ersichtlich, dass schon eine moderate mittlere Anzahl assoziierter vertikaler Kanten die Navigation in kritischen Lokalisierungsszenarien wie lange Korridore entscheidend verbessern kann. Ausserdem kann die Anforderung, dass die Operationsumgebung fortlaufend Merkmale eines bestimmten Types aufweisen muss, im Interesse robuster Navigation gelockert werden. Eine Verbesserung der Positionsschätzung im allgemeinen und besonders in der Orientierung wurde festgestellt, wobei die absoluten Werte eine hohen Lokalisierungsgenauigkeit belegen.

Ausserdem konnte demonstriert werden, dass die Lokalisierung mit den Sensoren Laser und Video eine Implementation auf einem vollständig autonomen Roboter in Echtzeit zulässt und zu praktikablen Zykluszeiten führt.

Weiterführende Arbeiten werden sich auf die Implementation fortlaufender Lokali- 
sierung während der Fahrt (on-the-fly) konzentrieren. Überdies scheint die Anwendung elaboriertere Assoziationsstrategien wie diejenigen von [6] und [10] vielversprechend, nicht nur im Falle der vertikalen Kanten, sondern auch für Liniensegmente.

\section{Referenzen}

[1] Arras K.O., Siegwart R.Y., "Feature Extraction and Scene Interpretation for Map-Based Navigation and Map Building", Proc. of SPIE, Mobile Robotics XII, Vol. 3210, p. 42-53, 1997.

[2] Arras K.O., Tomatis N., "Improving Robustness and Precision in Mobile Robot Localization by Using Laser Range Finding and Monocular Vision", Proc. of the Third European Workshop on Advanced Mobile Robots (Eurobot 99), p. 17785, Zurich, Switzerland, Sept. 6-9, 1999.

[3] Kaiser O., P?ffner R., Vestli S., Astolfi A., "Positionsregelung für nicht-holonome mobile Roboter”, 11. Fachgespräche Autonome Mobile Systeme (AMS'95), Germany, 1995.

[4] Bar-Shalom Y., Fortmann T.E., Tracking and Data Association, Mathematics in Science and Engineering, Vol. 179, Academic Press Inc., 1988.

[5] Brega R., "A Real-Time Operating System Designed for Predictability and RunTime Safety”, Proc. of the Fourth Int. Conference on Motion and Vibration Control (MOVIC'98), p. 379-84, Zurich, Switzerland, 1998.

[6] Castellanos J.A., Mobile Robot Localization and Map Building: A Multisensor Fusion Approach, Doctoral Thesis, Departamento de Informática e Ingeniería de Sistemas, Universidad de Zaragoza, Spain, May 1998.

[7] Chenavier F., Crowley J.L., "Position Estimation for a Mobile Robot Using Vision and Odometry", Proc. of the 1992 IEEE Int. Conference on Robotics and Automation, p. 2588-93, Nice, France, 1992.

[8] Crowley J.L., "World Modeling and Position Estimation for a Mobile Robot Using Ultrasonic Ranging," Proc. of the 1989 IEEE Int. Conference on Robotics and Automation, p. 674-80, Scottsdale, AZ, 1989.

[9] Leonard J.J., Durrant-Whyte H.F., Directed Sonar Sensing for Mobile Robot Navigation, Kluwer Academic Publishers, 1992.

[10] Muñoz A.J., Gonzales J., “Two-Dimensional Landmark-based Position Estimation from a Single Image”, Proc. of the 1998 IEEE Int. Conference on Robotics and Automation, Leuven, Belgium, 1998, p. 3709-14.

[11] Neira J., Tardos J.D., Horn J., Schmidt G., "Fusing Range and Intensity Images for Mobile Robot Localization," IEEE Transactions on Robotics and Automation, 15(1):76-84, 1999.

[12] Pérez J.A., Castellanos J.A., Montiel J.M.M., Neira J. and Tardós J.D., "Continuous Mobile Robot Localization: Vision vs. Laser," Proc. of the 1999 IEEE Int. Conference on Robotics and Automation, Detroit, USA, 1999. 